\title{
UTILIZAÇÃO DO TRATAMENTO CONSERVADOR DA FISIOTERAPIA EM INDIVIDUOS COM LESÃO DO LIGAMENTO COLATERAL LATERAL: UMA REVISÃO NARRATIVA
}

\author{
REFLECTIONS AND RESULTS PNI FROM ITS IMPLEMENTATION TO THE PRESENT
}

\begin{abstract}
Erika Naianny da Silva Mascarenhas ${ }^{1}$, Enio kaundson Fausto Soares ${ }^{1}$; Francisco Rubson bezerra de lima ${ }^{1}$, Joelma Gomes da Silva ${ }^{1}$, Jaiza Marques Medeiros e Silva ${ }^{1}$, Oziel Tardely Sousa farias ${ }^{1}$, Lucas Ewerton Rodrigues Gomes ${ }^{1}$, Elanny Mirelle da Costa $^{1}$
\end{abstract}

${ }^{1}$ Faculdade Nova Esperança de Mossoró (FACENE/RN)

Info

Recebido: 07/2020

Publicado: $10 / 2021$

DOI: $10.37951 / 2358-260 X .2021 v 8 \mathrm{i} 2.5892$

ISSN: 2358-260X

Palavras-Chave
Lesão de Ligamento; Ligamento Colateral;
Ruptura do Ligamento; Ligamento
colateral lateral; Ligamento.
Keywords:
Ligament Injury; Collateral Ligament;
Ligament rupture; Lateral collateral
ligament; Ligament.

\begin{abstract}
Resumo
O ligamento colateral lateral (LCL), tem como função principal a estabilização dinâmica da articulação tibiofemoral. Mediante a sua biomecânica e anatomia, o LCL localiza-se póstero-lateralmente em relação ao joelho e não possui alta incidência de lesão se comparado aos demais ligamentos. Dessa forma, o tratamento conservador da Fisioterapia torna-se um grande aliado para a recuperação da estabilidade e retorno às atividades de vida diárias sem submeter-se aos riscos de cirurgias. Objetivo: Buscar na literatura estudos sobre a eficácia do tratamento conservador fisioterapêutico na estabilidade e funcionalidade do joelho nas lesões de LCL em pacientes adolescentes e adultos. Materiais e Métodos: Trata-se de uma revisão narrativa onde as buscas foram realizadas nas bases de dados Scielo, Pubmed e Science Direct publicados no período de 2011 a 2020 escritos em Inglês; Espanhol e Português disponíveis na íntegra de maneira gratuita. Foram inclusos artigos completos e incompletos que abordassem o tratamento conservador da Fisioterapia na recuperação da estabilidade articular do joelho e seus correspondentes. Já os critérios
\end{abstract} de exclusão deram-se por cartas ao editor, monografias e amostras editoriais. Resultados e Discussão: Foram selecionados 11 artigos no total, onde somente 6 preencheram os critérios de inclusão estabelecidos. Os estudos mostram que no tratamento foram implementados programas de exercícios funcionais focados na região posterolateral afim de trabalhar o fortalecimento, promover analgesia e movimentação ativa do membro afetado. Visando melhorias na instabilidade do joelho e consequentemente a funcionalidade na deambulação. Ainda existem poucos estudos na literatura no que se refere a atuação da fisioterapia no tratamento de lesões no ligamento colateral lateral em quaisquer que seja o nível. É de grande valia esse conhecimento para estudantes e profissionais da área, uma vez que é possível contribuir com a comunidade acadêmica e desenvolvimento de profissionais mais qualificados. Conclusão: Portanto, o tratamento conservador da Fisioterapia promoveu melhoras significativas que auxiliaram na devolução da estabilidade articular e funcionalidade desses indivíduos favorecendo o retorno das atividades diárias com sucesso. 


\section{INTRODUÇÃO}

Anatomicamente o Ligamento Colateral Lateral localiza-se na porção lateral joelho e insere-se na cabeça da fíbula. Quanto a sua biomecânica é responsável pela estabilização lateral do joelho, pois resiste as forças da articulação tibiofemoral evitando o varismo em excesso em conjunto com outros ligamentos (Cottini e Weigert, 2011).

Segundo Haddad et al (2016); o ligamento colateral lateral (LCL), também conhecido complexo póstero-lateral ou canto póstero-lateral tem como função principal a estabilização dinâmica da articulação tíbiofemoral, essa estrutura contribui para uma considerável resistência em relação ao estresse em varo e, consequentemente, auxilia na efetividade da rotação externa do membro, quando encontra-se em sua total integridade. O LCL faz parte do grupo dos tecidos moles presentes no corpo humano e por isso é considerado como parte das estruturas que estão menos suscetíveis a lesões presentes na articulação do joelho.

De acordo com Kramer et al. (2020), as lesões LCL correspondem a um percentual inferior quando se trata dos danos causados nessa estrutura, sendo considerada de baixa incidência em comparação a outros ligamentos. Esse tipo de lesão está comumente associado ao esporte como também às lesões por acidentes de trânsito. Os traumas decorrentes desse ligamento, envolvem uma alta energia lesionando a articulação tíbio-femoral que no momento é hiperestendida resultando em uma força no varismo acometendo o LCL, (Davenport D et al.,2018).

Mediante a isso, o tratamento conservador da Fisioterapia torna-se um grande aliado na recuperação da estabilidade e retorno as atividades de vida diárias sem que esses indivíduos se submetam aos riscos dos procedimentos cirúrgicos. Assim é visto na literatura que a reabilitação com enfoque no fortalecimento muscular da região subjacente, aprimoramento do controle neuromuscular, equilíbrio e propriocepção; de maneira geral vai auxiliar na recuperação mais rápida desses indivíduos (Haddad et al.,2016).

Contudo, esse tipo de lesão traumática decorrente do ligamento colateral lateral é considerado incomum, principalmente em adolescentes pois, existem poucas orientações na literatura a respeito desse tipo de dano ligamentar em específico e, há ainda uma escassa contribuição a respeito de tratamentos conservadores, prognóstico e manejo fisioterapêutico (Haddad et al.,2016). Assim, o objetivo deste artigo foi buscar na literatura estudos sobre a eficácia do tratamento conservador fisioterapêutico relacionados a instabilidade do joelho nas lesões de ligamento colateral lateral (LCL) em pacientes adolescentes e adultos.

\section{MATERIAIS E MÉTODOS}

Esse estudo trata-se de uma revisão narrativa, onde as buscas foram realizadas nas bases de dados Scientific Electronic Library Online (SCIELO), Pubmed e ScienceDirect publicados no período de 2011 a 2020 escritos em Inglês; Espanhol e Português disponíveis na íntegra com livre acesso. Como critérios de inclusão, foram selecionados artigo completos que abordassem os tratamentos conservadores da Fisioterapia na recuperação da estabilidade articular do joelho, funcionalidade e seus correspondentes, e escritos na Língua Portuguesa e Língua Inglesa. Já os critérios de exclusão deram-se por cartas ao editor, monografias e amostras editoriais e que estivessem fora do período de publicação escolhido.

Os artigos foram selecionados por pares às cegas, onde inicialmente foi realizada a leitura a partir dos títulos e resumos, que atendessem a necessidade da pesquisa e posteriormente, foi realizada a leitura dos artigos completos. Os resultados foram compartilhados 
entre os autores tendo como base os critérios de inclusão e exclusão. Após essa etapa foi realizada a leitura criteriosa dos artigos pelos revisores.

\section{RESULTADOS E DISCUSSÃO}

Nas buscas para a construção deste estudo foram encontrados no total de 16 artigos, dentre esses, 10 foram localizados na PubMed; 4 no Science Direct e 2 na Scielo, onde a partir disso foi realizada uma leitura minuciosa entre os autores para aplicar os critérios de inclusão. Dentre os 16, haviam 5 artigos que tratava de abordagens cirúrgicas e manejo exclusivo de condutas médicas, restando assim, 11 artigos ao total em que somente 6 atendiam as necessidades dos critérios de inclusão. Foram excluídos os artigos que não haviam resultados quanto ao manejo conservador das lesões de LCL. Durante a leitura entre os autores, 2 artigos foram excluídos por possuir estritamente a intervenção cirúrgica para a recuperação da lesão; 1 artigo por estar fora do ano de publicação estabelecido; outro por estar em duplicata e o último artigo excluído por se tratar de uma monografia e também pelo ano de publicação.

Mediante os artigos selecionados, Davenport et al (2018), aponta que inicialmente em sua abordagem terapêutica foi utilizado a analgesia juntamente com uso da órtese de joelheira articulada seguindo um plano terapêutico com exercícios ativos de flexão e extensão respeitando a progressão do lesado, permitido realizar tais movimentos para a melhora da ADM, o que se faz necessário e importante na movimentação de uma articulação e sua funcionalidade.

Nos achados de Schweller et al. (2021), foi abordado que em casos de edema nas regiões subjacentes a lesão pode ser feita uma liberação miofascial de forma indireta que propositalmente irá promover drenagem linfática local ademais, que para a melhoria da ADM pode ser utilizado o método de pressão no sentido oposto na parte posterior a lesão, o que melhoraria a funcionalidade e qualidade de vida dos indivíduos, corroborando assim com os estudos de Davenport et (2018).

No trabalho de Haddad et al. (2016), foi avaliada a progressão dos pacientes de acordo com o andamento da terapêutica para verificar e qualificar a instabilidade do joelho em relação a marcha. Durante a terapêutica foi possível obter o ganho de força controle neuromuscular, equilíbrio e propriocepção com treinos progressivos de acordo com as melhorias dos indivíduos, tendo como objetivo principal o fortalecimento funcional, com a utilização exercícios físicos e testes para fortalecer joelho e músculos envolvidos, promovendo a analgesia e estabilidade articular, e consequentemente a funcionalidade na deambulação e de movimentos dependentes da articulação (Haddad et al.,2016).

Mediante a isso, Kramer et al. (2020), nota-se que há uma raridade das lesões do LCL, por isso elas geralmente ocorrem posteriormente a outras lesões ligamentares mais comumente apontadas na literatura como lesões de ligamento colateral medial, cruzado posterior e afins que geram uma vulnerabilidade patelar maior; dentro desse contexto também foi avaliado que o comprometimento do LCL acontece em maior número no futebol. Embora as lesões de maior impacto na estrutura e acometimento do LCL sejam no futebol, há uma incidência de casos em quadros de obesidade onde a lesão na parte lateral do joelho também pode ocorrer com a energia imposta pelo próprio peso corporal (Cottini e Weigert, 2011).

Os estudos mencionados anteriormente trazem abordagens que se correlacionam, o que vai em contrapartida ao estudo de Kennedy et al. (2018) no qual relata que o complexo póstero-lateral quando lesado possui uma recuperação ruim e paralelo a isso o manejo conservador têm causado uma instabilidade recorrente no joelho. Apesar da negativa dos autores, 
logo adiante os mesmos afirmam que embora cause instabilidade no joelho, ainda assim o tratamento tradicional é preferencialmente utilizado em casos de lesões no PCL pois recupera mais rápido o movimento da estrutura, proporcionando ganhos clínicos mais significativos e com menores restrições.

Portanto, foi possível ainda constatar que a literatura é muito escassa, e existem poucas evidências quando se trata de LCL, se comparadas a ligamento cruzado anterior (LCA) e ligamento cruzado posterior (LCP) que são bem mais enfatizadas nas pesquisas de bases de dados. Necessitando de mais estudos de tal patologia para se aprimorar os conhecimentos e manejo da fisioterapia no tratamento conservador.

\section{CONCLUSÃO}

Apesar da escassez em estudos relacionados ao tema, foi observado através dos estudos selecionados que o tratamento conservador da fisioterapia contribuiu de forma significativa, sendo bastante eficaz para a reabilitação da lesão do Ligamento Colateral Lateral (LCL), possibilitando assim a retomada da estabilidade articular e capacidade funcional, equilíbrio e propriocepção dos indivíduos, juntamente com o retorno ativo de suas atividades diárias. Porém, ainda se faz necessário mais estudos sobre a relação tratamento fisioterapêutico conservador e a Lesão do Ligamento Colateral Lateral, visto que são poucos estudos atualizados sobre o assunto, o que dificulta uma abordagem mais ampla sobre a temática.

\section{REFERÊNCIAS}

Kramer DE, Miller PE, Berrahou IK, Yen YM, Heyworth BE. Collateral Ligament Knee Injuries in Pediatric and Adolescent Athletes. J Pediatr Orthop. 2020 Feb;40(2):71-77. Disponível em: http//doi/10.1097/BPO.0000000000001112. PMID: 31923166. Acesso em: 29 jun. 2021.
Haddad MA, Budich JM, Eckenrode BJ. CONSERVATIVE MANAGEMENT OF AN ISOLATED GRADE III LATERAL COLLATERAL LIGAMENT INJURY IN AN ADOLESCENT MULTI-SPORT ATHLETE: A CASE REPORT. Int J Sports Phys Ther. 2016 Aug;11(4):596-606. Disponível em: PMID: 27525183; PMCID: PMC4970849. Acesso em: 29 jun. 2021.

Davenport D, Arora A, Edwards MR. Non-operative management of an isolated lateral collateral ligament injury in an adolescent patient and review of the literature. BMJ Case Rep. 2018 May 15;2018:bcr2017223478. Disponível em: http//doi/10.1136/bcr-2017-223478. PMID: 29764846; PMCID: PMC5965770. Acesso em: 29 jun. 2021.

Schweller EW, Ward PJ. Posterolateral Corner Knee Injuries: Review of Anatomy and Clinical Evaluation. J Am Osteopath Assoc. 2015 Dec;115(12):725-31. Disponível em: http//doi/10.7556/jaoa.2015.148. PMID: 26618818. Acesso em: 19 jul. 2021.

COTTINI, Lucas; WEIGERT, Guilherme. Lesão dos Ligamentos Colaterais do Joelho. Jaleko Artmed, 2011. [Acesso em 21 jul. 2021]. Disponível em: https://jaleko-files.s3-saeast1.amazonaws.com/apostilaweb/5f74e3eeda e $7 \mathrm{~b} \_L e s \%$ C3 $\%$ A30 $\% 20$ dos $\% 20$ Ligamentos $\% 2$ 0Colaterais $\% 20$ do $\% 20$ Joelho $\% 20(1)$.pdf.

Kennedy MI, Bernhardson A, Moatshe G, Buckley PS, Engebretsen L, LaPrade RF. Fibular Collateral Ligament/ Posterolateral Corner Injury: When to Repair, Reconstruct, or Both. Clin Sports Med. 2019 Apr;38(2):261-274. Disponível em: http://doi/ 10.1016/j.csm.2018.11.002. Epub 2019 Jan 19. PMID: 30878048. Acesso em: 21 jul. 2021. 